\title{
Lista de esponjas marinas asociadas al arrecife Tuxpan, Veracruz, México
}

\section{Checklist of marine sponges from Tuxpan Reef, Veracruz, Mexico}

\author{
Carlos González-Gándara*, Abelardo Patiño-García ${ }^{1}$, Uribe Asís-Anastasio ${ }^{1}$, Arturo Serrano ${ }^{1}$ y Patricia \\ Gómez ${ }^{2}$ \\ ${ }^{I}$ Laboratorio de Arrecifes Coralinos, Escuela de Biología, Facultad de Ciencias Biológicas y Agropecuarias, Campus Tuxpan. Universidad Veracruzana. \\ Carr. Tuxpan-Tampico Km 7.5, 92850 Tuxpan, Veracruz, México. \\ ${ }^{2}$ Laboratorio de Sistemática y taxonomía de esponjas marinas, Instituto de Ciencias del Mar y Limnología, Universidad Nacional Autónoma de \\ México. Circuito exterior s/n, Ciudad Universitaria, 04510 México, D. F., México. \\ *Correspondencia: cggandara@hotmail.com
}

\begin{abstract}
Resumen. Se presenta la lista de esponjas marinas (Porifera: Demospongiae) del arrecife Tuxpan, Veracruz, México, colectadas en 2004, 2005 y 2006 mediante buceo libre y con equipo autónomo SCUBA. Los resultados muestran la presencia de 18 especies pertenecientes a 13 géneros y 13 familias, 17 de estas especies son nuevos registros para los arrecifes coralinos del norte de Veracruz y una (Aplysina cauliformis Carter, 1882) para el estado. La información puede auxiliar para definir las estrategias de manejo, monitoreo y protección de estas formaciones arrecifales que recientemente han sido propuestas como área de protección de flora y fauna.
\end{abstract}

Palabras clave: esponjas marinas, Porifera, arrecifes coralinos, Veracruz, México.

\begin{abstract}
A checklist of marine sponge species (Porifera: Demospongiae) from Tuxpan reef, Veracruz, Mexico, collected during 2004, 2005 and 2006 by free and SCUBA diving equipment, is presented. The results show the presence of 18 species belonging to 13 genera and 13 families. 17 speices represent new records for the northern coral reefs of Veracruz, and the $18^{\text {th }}$ species (Aplysina cauliformis Carter, 1882) is a new record for the state. This information may help to define appropriate management, monitoring and protection strategies for the coral reefs of the north of Veracruz, which have been proposed as a natural preserve area recently.
\end{abstract}

Key words: marine sponges, Porifera, Coral reefs, Veracruz, Mexico.

\section{Introducción}

El escaso conocimiento de los ecosistemas marinos y sus componentes bióticos conduce a la toma de decisiones basadas en supuestos, lo que puede fomentar la pérdida de diversidad biológica y el desequilibrio en el funcionamiento de los ecosistemas (Carricart-Ganivet y Beltrán-Torres, 1997). La heterogeneidad ambiental típica de los arrecifes coralinos permite el desarrollo de numerosas comunidades bióticas. Una de éstas es la de esponjas marinas, la cual presenta una mayor diversidad que los corales mismos (Díaz y Rützler, 2001; Wullf, 2006). Los miembros del phylum Porifera llevan a cabo funciones clave que ayudan al desarrollo de las formaciones arrecifales, dado que unen el marco estructural del arrecife (Wullf y Buss, 1979; Wullf, 2006). Por otro lado, son activos filtradores de bacterias y

Recibido: 05 marzo 2007; aceptado: 06 julio 2008 materia orgánica disuelta aumentando la claridad del agua, actúan en procesos de bioerosión del material calcáreo produciendo nuevas áreas de fijación y funcionan como hospederos de muchos invertebrados y peces (Díaz et al., 1985; Díaz y Rützler, 2001; Reis y Leao, 2000). Algunos autores han planteado la presencia y abundancia de algunas especies, o su diversidad como indicadores de estrés en ecosistemas marinos por su estrecha relación con el medio (Alcolado y Herrera, 1987; Carballo et al., 1996; 2004).

Los estudios realizados en los arrecifes coralinos del centro de Veracruz, indican la presencia de 50 especies de esponjas (Green, 1977; Green et al., 1986; López-Herrera, 1992; Gómez, 2002, 2007). En los arrecifes coralinos del norte de Veracruz, sólo se ha observado Neopetrosia subtriangularis en el arrecife Lobos (Chávez et al., 1970). El presente trabajo constituye el primer registro de especies del phylum Porifera en la región, particularmente para el arrecife Tuxpan. 


\section{Materiales y métodos}

La presente investigación se realizó en el arrecife Tuxpan, ubicado al norte de Veracruz, aproximadamente a $300 \mathrm{~km}$ del Sistema Arrecifal Veracruzano (SAV que geográficamente se ubica a $21^{\circ} 01^{\prime} \mathrm{N}$ y $97^{\circ} 11^{\prime} \mathrm{O}$ (Fig. 1). La observación y recolecta de esponjas se realizó en 3 periodos: septiembre-octubre de 2004, abril-junio de 2005 y mayo-octubre de 2006, mediante buceo libre y con equipo autónomo SCUBA, utilizando un cuchillo para la recolecta. Se anotaron datos de forma, tamaño, coloración consistencia, características y distribución de los ósculos. Posteriormente, las esponjas se fijaron en formol comercial al $10 \%$ disuelto en agua marina y después se pasaron a una solución de alcohol etílico al 70\%. En el laboratorio se realizaron cortes gruesos para observar los detalles de la estructura esqueletal siguiendo los criterios de Gómez (2002) y Carballo et al. (2004). Los organismos fueron depositados en la colección del Laboratorio de Arrecifes Coralinos de la Universidad Veracruzana (UVE) en Tuxpan, Veracruz.

\section{Resultados}

Se identificó un total de 18 especies pertenecientes a 13 géneros y 13 familias. En el Cuadro 1, se citan las especies ordenadas según el criterio de Hooper y van Soest

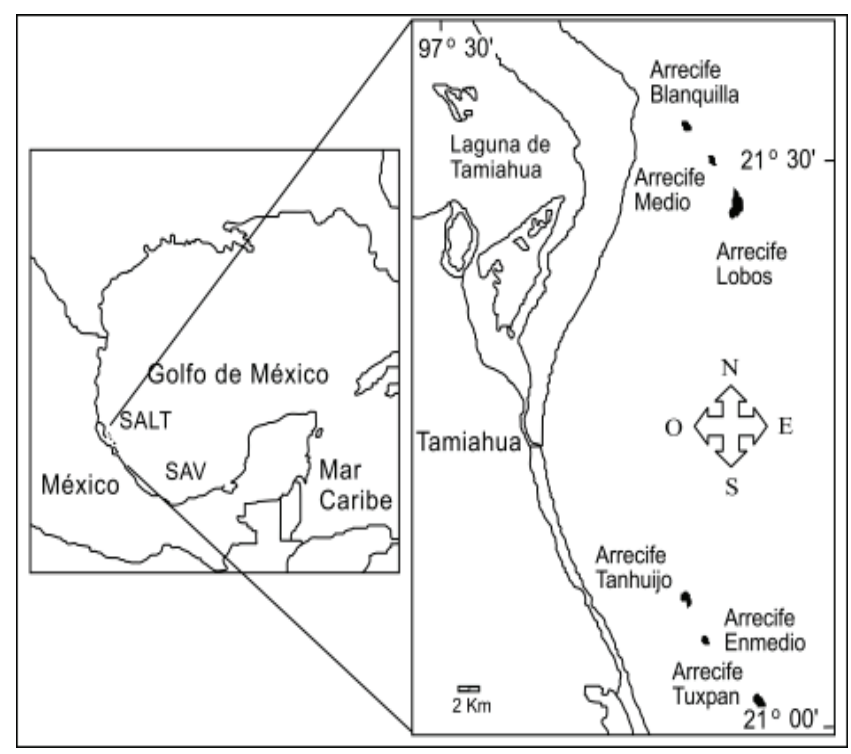

Figura 1. Localización geográfica del arrecife Tuxpan. SALT= Sistema Arrecifal Lobos-Tuxpan. SAV=Sistema Arrecifal Veracruzano.
(2002). De las 18 especies listadas, 17 se citan por primera vez para los arrecifes coralinos de la región y 1 para los ecosistemas arrecifales de Veracruz. El nuevo registro, Aplysina cauliformis (Carter, 1882), es una especie ramosa rastrera de consistencia firme pero elástica, presenta prolongaciones de hasta $50 \mathrm{~cm}$ de longitud y diámetro de $5 \mathrm{~mm}$ a $1 \mathrm{~cm}$. Su color en vivo es amarillo que se torna castaño claro al preservarlo. Su esqueleto presenta fibras estratificadas, meduladas de color ámbar con 75 a $170 \mu$ de diámetro, la médula alcanza del 15 al 35\% del diámetro de la fibra, abarcando mayor longitud al adelgazarse la fibra hacia la superficie. El esqueleto forma una retícula poligonal y alargada en algunas partes, con malla de 450 a $690 \mu \mathrm{m}$. Los ejemplares de esta especie fueron colectados a $6 \mathrm{~m}$ de profundidad en un ambiente de corales masivos rodeados de sustrato arenoso-limoso, ubicado en la pendiente de sotavento.

\section{Discusión}

La lista de esponjas marinas que se presenta en este trabajo constituye el primer registro formal del phylum Porifera para los arrecifes coralinos del norte de Veracruz e incluye un nuevo registro (A. cauliformis) para el estado. Esta especie se puede confundir fácilmente con especies similares como Aplysina archeri y Aplysina fulva, tanto en la forma como en el color, aun en cualquiera de los ambientes en los que coexisten. Sin embargo, la forma y el color de $A$. cauliformis siempre tienden a ser característicos. Aplysina fulva se torna negra totalmente o castaño negruzco al preservarla y $A$. cauliformis no lo hace. Por su parte, $A$. archeri tiene un parecido con $A$. cauliformis por las prolongaciones ramosas o fistulares que parten de su forma tubular característica, pero esta última es de color lila y se torna castaño (Wiedenmayer, 1977; van Soest, 1978; Zea, 1987).

El color en vivo de $A$. cauliformis es variable, podemos encontrar desde el amarillo oscuro al amarillo castaño con tintes verdosos, morado-rosáceos, azul-liláceos (Wiedenmayer, 1977), amarillo claro a oscuro con tonos salmón y rosa carne (Zea, 1987). En preservado se registran colores castaño rojizo (Wiedenmayer, 1977), rojizo, violeta (Zea, 1987), castaño rosáceo (van Soest, 1978) y castaño claro. Sin embargo, en organismos preservados la coloración no puede tomarse como determinante en la definición de las especies, puesto que se adquiere de acuerdo al método de fijación utilizado. En algunas especies pertenecientes al género Aplysina, es posible afirmar que el carácter aerofóbico de éstas (cambio del color al contacto con el aire) las agrupa en este género, con sus excepciones. El caso de $A$. cauliformis es una excepción y 
Cuadro 1. Lista de las esponjas de la clase Demospongiae, registradas en el arrecife Tuxpan, Veracruz, México. Las especies fueron ordenadas siguiendo el criterio de Hooper y van Soest (2002). Georreferencias: ${ }^{1)} 21^{\circ} 01^{\prime} 375^{\prime \prime}$ N; $97^{\circ} 11^{\prime} 347^{\prime \prime} \mathrm{O}$; $^{2} 2^{\circ} 1^{\circ} 01^{\prime} 338^{\prime \prime} \mathrm{N}$;

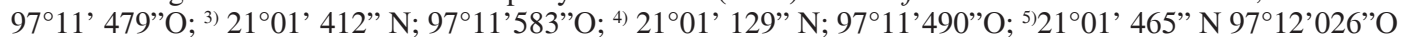

\begin{tabular}{|c|c|c|c|c|c|c|c|}
\hline Orden & Familia & Género & Especie & Referencia & Hábitat & $\begin{array}{l}\text { Prof. } \\
\text { (m) }\end{array}$ & $\begin{array}{l}\text { Núm. de } \\
\text { catálogo }\end{array}$ \\
\hline \multirow[t]{2}{*}{ Hadromerida } & \multirow[t]{2}{*}{ Clionaidae } & \multirow[t]{2}{*}{ Cliona } & $\begin{array}{c}\text { Cliona caribbaea } \\
\text { Carter1882 }\end{array}$ & $\begin{array}{l}\text { Rützler, (1974); } \\
\text { Gómez (2002); } \\
\text { Schönberg } \\
\text { (2000) }\end{array}$ & $\begin{array}{c}\text { Sustrato calcáreo } \\
\text { con Echinometra } \\
\text { lucunter }^{1}\end{array}$ & $1 \mathrm{~m}$ & UVEOO3 \\
\hline & & & $\begin{array}{l}\text { Cliona delitrix } \\
\text { (Pang 1973) }\end{array}$ & $\begin{array}{l}\text { Pang (1973); } \\
\text { Gómez (2002, } \\
\text { 2007) }\end{array}$ & Corales masivos ${ }^{3}$ & $6 \mathrm{~m}$ & UVEOO1 \\
\hline Chondrosida & Chondrillidae & Chondrilla & Chondrilla sp. & $\begin{array}{l}\text { Hooper y van } \\
\text { Soest (2002) }\end{array}$ & $\begin{array}{c}\text { Sustrato calcáreo } \\
\text { con Echinometra } \\
\text { lucunter }^{1}\end{array}$ & $1 \mathrm{~m}$ & UVEOO4 \\
\hline \multirow[t]{5}{*}{ Poecilosclerida } & Coelosphaeridae & Lissodendoryx & $\begin{array}{c}\text { Lissodendoryx } \\
\text { isodictyalis (Carter } \\
\text { 1882) }\end{array}$ & $\begin{array}{l}\text { van Soest } \\
\text { (1984); Green } \\
\text { et al.(1986); } \\
\text { Gómez (2002) }\end{array}$ & Pastos marinos ${ }^{2}$ & $0.6 \mathrm{~m}$ & UVEOO9 \\
\hline & Desmacididae & Desmapsamma & $\begin{array}{c}\text { Desmapsamma } \\
\text { anchorata } \\
\text { Carter1882 }\end{array}$ & $\begin{array}{l}\text { Green et al. } \\
\text { (1986); Gómez } \\
\text { (2002) }\end{array}$ & Pastos marinos $^{2}$ & $0.6 \mathrm{~m}$ & UVE010 \\
\hline & Iotrochotidae & Iotrochota & $\begin{array}{c}\text { lotrochota } \\
\text { birotulata (Higgin } \\
1877)\end{array}$ & $\begin{array}{c}\text { Green et al. } \\
\text { (1986); Hooper } \\
\text { y van Soest } \\
\text { (2002); Gómez } \\
\text { (2002) }\end{array}$ & Corales masivos ${ }^{4}$ & $6 \mathrm{~m}$ & UVEOO5 \\
\hline & Crambeidae & Monanchora & $\begin{array}{c}\text { Monanchora } \\
\text { arbuscula } \\
\text { (Duchassaing y } \\
\text { Michelotti 1864) }\end{array}$ & $\begin{array}{c}\text { van Soest, } \\
\text { (1984); Gómez } \\
\text { (2002) }\end{array}$ & Corales masivos $^{5}$ & $9 \mathrm{~m}$ & UVE012 \\
\hline & Raspailiidae & Ectyoplasia & $\begin{array}{c}\text { Ectyoplasia ferox } \\
\text { Duchassaing y } \\
\text { Michelotti } 1864\end{array}$ & $\begin{array}{l}\text { Gómez (2002, } \\
\text { 2007) }\end{array}$ & Corales masivos ${ }^{4}$ & $6 \mathrm{~m}$ & UVE014 \\
\hline \multirow[t]{5}{*}{ Haplosclerida } & Callyspongiidae & Callyspongia & $\begin{array}{c}\text { Callyspongia } \\
\text { vaginalis (Lamarck } \\
1814 \text { ) }\end{array}$ & $\begin{array}{l}\text { Green et al. } \\
\text { (1986); Gómez } \\
\text { (2002) }\end{array}$ & Corales masivos ${ }^{4}$ & $6 \mathrm{~m}$ & UVEOO2 \\
\hline & \multirow{3}{*}{ Niphatidae } & \multirow{3}{*}{ Amphimedon } & $\begin{array}{c}\text { Callyspongia } \\
\text { armigera } \\
\text { (Duchassaing y } \\
\text { Michelotti 1864) }\end{array}$ & $\begin{array}{c}\text { Green et al. } \\
\text { (1986); Hooper } \\
\text { y van Soest } \\
\text { (2002); Gómez } \\
\text { (2002) }\end{array}$ & Corales masivos ${ }^{4}$ & $6 \mathrm{~m}$ & UVE018 \\
\hline & & & $\begin{array}{l}\text { Amphimedon } \\
\text { compressa } \\
\text { Duchassaing y } \\
\text { Michelotti } 1864\end{array}$ & $\begin{array}{l}\text { Hooper y van } \\
\text { Soest (2002); } \\
\text { Gómez (2002) }\end{array}$ & Corales masivos ${ }^{4}$ & $6 m$ & UVEOO6 \\
\hline & & & $\begin{array}{c}\text { Amphimedon viridis } \\
\text { Duchassaing y } \\
\text { Michelotti } 1864\end{array}$ & $\begin{array}{l}\text { Green et al. } \\
\text { (1986) }\end{array}$ & $\begin{array}{c}\text { Sustrato calcáreo } \\
\text { con Echinometra } \\
\text { lucunter }\end{array}$ & $1 \mathrm{~m}$ & UVEOO7 \\
\hline & Petrosidae & Neopetrosia & $\begin{array}{c}\text { Neopetrosia } \\
\text { subtriangularis } \\
\text { (Duchassaing 1850) }\end{array}$ & $\begin{array}{l}\text { Green et al. } \\
\text { (1986); Gómez } \\
\text { (2002) }\end{array}$ & Corales masivos ${ }^{5}$ & $9 \mathrm{~m}$ & UVE019 \\
\hline Dictyoceratida & Spongiidae & Hyrtios & $\begin{array}{l}\text { Hyrtios proteus } \\
\text { Duchassaing y } \\
\text { Michelotti } 1864\end{array}$ & Zea (1987) & $\begin{array}{c}\text { Sustrato calcáreo } \\
\text { con Echinometra } \\
\text { lucunter }\end{array}$ & $1 \mathrm{~m}$ & UVE013 \\
\hline
\end{tabular}


Cuadro 1. Continúa

\begin{tabular}{|c|c|c|c|c|c|c|c|}
\hline Orden & Familia & Género & Especie & Referencia & Hábitat & $\begin{array}{l}\text { Prof. } \\
\text { (m) }\end{array}$ & $\begin{array}{l}\text { Núm. de } \\
\text { catálogo }\end{array}$ \\
\hline \multirow{4}{*}{ Verongida } & \multirow[t]{2}{*}{ Irciniidae } & \multirow[t]{2}{*}{ Ircinia } & $\begin{array}{c}\text { Ircinia felix } \\
\text { (Duchassaing y } \\
\text { Michelotti 1864) }\end{array}$ & $\begin{array}{l}\text { van Soest (1978) y } \\
\text { Gómez (2002) }\end{array}$ & Corales masivos $^{3}$ & $6 \mathrm{~m}$ & UVE017 \\
\hline & & & $\begin{array}{l}\text { Ircinia strobilina } \\
\text { (Lamarck 1816) }\end{array}$ & $\begin{array}{l}\text { Green et al. (1986); } \\
\text { Gómez (2002) }\end{array}$ & Corales masivos ${ }^{5}$ & $9 \mathrm{~m}$ & UVEOO8 \\
\hline & \multirow[t]{2}{*}{ Aplysinidae } & \multirow[t]{2}{*}{ Aplysina } & $\begin{array}{l}\text { Aplysina fistularis } \\
\text { (Pallas 1766) }\end{array}$ & Gómez (2002) & Corales masivos ${ }^{3}$ & $6 \mathrm{~m}$ & UVE011 \\
\hline & & & $\begin{array}{l}\text { Aplysina cauliformis } \\
\text { (Carter 1882) }\end{array}$ & $\begin{array}{c}\text { Wiedenmayer (1977); } \\
\text { van Soest (1978); Zea } \\
\text { (1987); Gómez-López } \\
\text { y Green (1984) }\end{array}$ & Corales masivos ${ }^{3}$ & $6 \mathrm{~m}$ & UVE016 \\
\hline
\end{tabular}

nunca se torna de color negro o castaño negruzco, como es característico de A. fulva.

La riqueza de esponjas en el arrecife Tuxpan es muy parecida a la citada para los arrecifes del Sistema Arrecifal Veracruzano (Green, 1977; Green et al., 1986; López-Herrera, 1992; Gómez, 2002, 2007), donde se han registrado 50 especies (Gómez, 2002, 2007), de las cuales 14 son comunes para ambos sistemas (Cuadro 2). Los contrastes observados en el número de especies se

Cuadro 2. Especies de esponjas comunes para los arrecifes de Veracruz. SAV=Sistema Arrecifal Veracruzano

\begin{tabular}{lcc}
\hline \multicolumn{1}{c}{ Especie } & SAV & Tuxpan \\
\hline C. caribbaea & $*$ & $*$ \\
C. delitrix & & $*$ \\
Chondrilla sp. & $*$ & $*$ \\
L. isodictyalis & $*$ & $*$ \\
D. anchorata & $*$ & $*$ \\
I. birotulata & $*$ & $*$ \\
M. arbuscula & $*$ & $*$ \\
E. ferox & $*$ & $*$ \\
C. vaginalis & $*$ & $*$ \\
C. armigera & $*$ & $*$ \\
A. compressa & $*$ & $*$ \\
A. viridis & $*$ & $*$ \\
N. subtriangularis & $*$ & $*$ \\
H. proteus & & $*$ \\
I. felix & $*$ & $*$ \\
I. strobilina & $*$ & $*$ \\
A. fistularis & $*$ & \\
A. cauliformis & &
\end{tabular}

explican por las diferencias en el esfuerzo de muestreo, el cual ha sido mayor en el SAV, tanto en el espacio como en el tiempo; muy probablemente, la mayor superficie del SAV ofrece más disponibilidad de sustrato para la fijación de las esponjas. Por otra parte, las condiciones ambientales que operan en los diferentes sistemas arrecifales pueden generar una composición faunística diferencial.

Las especies registradas en este trabajo son las mismas que para el SAV y el Caribe, lo cual parece indicar que existe un transporte de larvas de esponjas desde el Caribe, lo que puede considerarse un indicador de la conexión ecológica (Jordán-Dahlgreen, 2002). El menor número de especies de esponjas observadas en el arrecife Tuxpan respecto al las del SAV sugiere un gradiente respecto al punto de origen, tal como ha sido documentado para las comunidades de octocorales entre el Caribe y el Golfo de México por Jordan-Dahlgreen (2002). Los sistemas arrecifales localizados en las costas del estado de Veracruz, al igual que el arrecife Tuxpan, están sujetos a una fuerte presión ambiental debido al creciente desarrollo portuario, de la industria petrolera y otras actividades humanas como la pesca y el turismo; sin embargo, el conocimiento de la diversidad biológica de estos ecosistemas aún es incipiente, particularmente para los arrecifes del norte de Veracruz. Por esto, los resultados de la presente investigación podrán servir como base para definir los planes de manejo y monitoreo que conduzcan a la protección de los arrecifes coralinos de Veracruz, mismos que recientemente han sido propuestos como área natural de protección de flora y fauna marina (Universidad Veracruzana, 2003).

\section{Agradecimientos}

Los autores agradecen a 2 árbitros anónimos que con 
sus comentarios permitieron mejorar el manuscrito. Las actividades de campo fueron financiadas parcialmente con recursos aportados por la Secretaría de Educación Pública a través del proyecto PIFI 3.3 2006-31-14 para el mejoramiento de la calidad del Programa de Biología, Zona Poza Rica Tuxpan.

\section{Literatura citada}

Alcolado, P. y A. Herrera.1987. Efectos de la contaminación sobre las comunidades de esponjas en el litoral de La Habana, Cuba. Reporte de Investigación. Instituto de Oceanología. Academia de Ciencias de Cuba 68:1-17

Carballo, J. L., J. A. Cruz-Barraza y P. Gómez. 2004. Taxonomy and description of clionaid sponges (Hadromerida, Clionaidae) from the Pacific Ocean of México. Zoological Journal of Linnean Society 141:353-397.

Carballo, J. L., S. A. Naranjo y J. C. García-Gómez. 1996. The use of marine sponges as stress indicators in marine ecosystems at Algeciras Bay (Southern Iberian Peninsula). Marine Ecology Progress Series 135:109-122.

Carricart-Ganivet, J. P. y A. Beltrán-Torres. 1997. Lista de corales pétreos (Hydrozoa: Milleporina, Stylasterina, Anthozoa: Scleractinia) de aguas someras del banco de Campeche, México. Revista de Biología Tropical 44:619-622.

Chávez, E. A, E. Hidalgo y M. Sevilla. 1970. Datos acerca de las comunidades bentónicas del arrecife Lobos, Veracruz. Revista de la Sociedad Mexicana de Historia Natural 31:211281.

Díaz, M. C., B. Álvarez y R. A Laughlin. 1985. The sponge fauna on a fringing coral reef in Venezuela 11: Community structure. In New perspective in sponge biology, K. Rutzler (ed.). Smithsonian Institution Press, Washington, D.C. p. 367-375.

Díaz, M. C. y K. Rützler. 2001. Sponges: an essential component of Caribbean coral reefs. Bulletin of Marine Sciences 69:535-546.

Gómez, P. 2002. Esponjas marinas del golfo de México y el Caribe. AGT, México, D. F. 134 p.

Gómez, P. 2007. Inventario de las esponjas del Parque Nacional Sistema Arrecifal Veracruzano, con nuevos registros de especies (Porifera: Demospongiae). In Investigaciones científicas en el Sistema Arrecifal Veracruzano, A. GranadosBarba, L. G. Abarca-Arenas y J. M. Vargas-Hernández. (eds.). Universidad Autónoma de Campeche, México. p. 51-72.

Gómez-López, P. y G. Green. 1984. Sistemática de las esponjas de Puerto Morelos, Quintana Roo, México. Anales del Instituto de Ciencias del Mar y Limnología. Universidad Nacional Autónoma de México 11:65-90

Green, G. 1977. Sinopsis taxonómica de trece especies de esponjas del arrecife La Blanquilla Veracruz, México. Anales del Instituto de Ciencias del Mar y Limnología, Universidad Nacional Autónoma de México 4:79-98.

Green, G., L. Fuentes y P. Gómez. 1986 Nuevos registros de Porifera del Arrecife La Blanquilla, Veracruz, México. Anales del Instituto de Ciencias del Mar y Limnología, Universidad Nacional Autónoma de México 13:273-300.

Hooper, J. N. A. y R. W. M. van Soest (eds.). 2002. Sysfema Porifera: A guide for fhe cIassification of sponges, vol. 1. Kluwer Academic, New York. 1101p.

Jordan-Dahlgreen, E. 2002. Gorgonian distribution patterns in coral reef environments of the Gulf of Mexico: evidence of sporadic ecological connectivity. Coral Reefs. 21:205215.

López-Herrera, M. 1992. Diagnosis taxonómica de las esponjas de las estructuras arrecifales de Antón Lizardo y Puerto de Veracruz. Tesis, Facultad de Ciencias, Universidad Nacional Autónoma de México. México, D. F. 88 p.

Pang, R. K. 1973. The systematics of some Jamaican excavating sponges. Postilla 161:1-75.

Reis, M. A. C. y Z. M. A. N. Leao. 2000. Bioerosion rate of the sponge Cliona celata (Grant 1826) from reefs in turbid waters, north Bahia, Brazil. Proceedings of $9^{\text {th }}$ International Coral Reef Symposium, Bali, M. K. Mossa, S. Soemodihardjo, A. Soegiarto, K. Romimohtarto, A. Nontji, Soekarno y Suharsono (eds.) 1: 273-278.

Rützler, K. 1974. The burrowing sponges of the Bermudas. Smithsonian Contributions to Zooology. 165:1-32.

Schönberg, C. H. L. 2000. Sponges of the 'Cliona viridis complex'- a key for species identification. Proceedings of $9^{\text {th }}$ International Coral Reef Symposium. Mossa, M. K., S. Soemodihardjo, A. Soegiarto, K. Romimohtarto y A. Nontji (eds.). Soekarno y Suharsono (eds.). Bali, 1:295-299.

Universidad Veracruzana. 2003. Documento técnico justificativo para la creación del área natural protegida Sistema Arrecifal Lobos-Tuxpam. Universidad Veracruzana, Xalapa, Veracruz. $52 \mathrm{p}$.

van Soest, R. W. M. 1978. Marine sponges from Curaçao and other Caribbean localities. Part I. Keratosa. Studies on the Fauna of Curaçao and other Caribbean Islands 179:1-94.

van Soest, R. W. M. 1984. Marine sponges from Curaçao and other Caribbean localities. Part III. Poecilosclerida. Studies on the fauna of Curaçao and other Caribbean Islands 99:1177.

Wiedenmayer, F. 1977. Shallow water sponges of the Western Bahamas. Birkhauser, Basel y Stuttgart. 278 p.

Wullf, J. L. 2006. Rapid diversity and abundance decline in a Caribbean coral reef sponge community. Biological Conservation 127:167-176.

Wullf, J. L. y L. W. Buss. 1979. Do sponges help hold coral reefs together? Nature. 281:474-475.

Zea, S. 1987. Esponjas del Caribe colombiano. Catálogo Científico. Colombia. 283 p. 